\title{
Exited configurations in the spectrum of five- times ionized indium (In VI)
}

\author{
E.Ya. Kononov ${ }^{1, *}$, R.R. Kildiyarova ${ }^{1}$, A.N. Ryabtsev ${ }^{1}$, A. Tauheed ${ }^{2}$, Swapnil $^{2}$, Hala $^{2}$, and \\ A. Saxena ${ }^{2}$ \\ ${ }^{1}$ Institute for Spectroscopy RAS, 108840 Troitsk, Moscow, Russia \\ ${ }^{2}$ Aligarh Muslim University, 202002 Aligarh, India
}

\begin{abstract}
An Indium spectrum was obtained in the region $160-230 \AA$ using the grazing-incidence spectrograph (incidence angle $85^{\circ}$, grating radius $3 \mathrm{~m}, 3600$ lines $/ \mathrm{mm}$ ) and a vacuum spark at peak currents $12-35$ kA. A preliminary identification of the $4 d^{8}-4 d^{7} 4 f$ and $4 d^{8}-4 d^{7} 5 f$ transitions in In VI have been done.
\end{abstract}

The most efficient radiation source for photo lithography on wavelength $135 \AA$ is a hightemperature plasma with multiply ionized atoms of tin. It is well established that radiative transitions of Sn IX - Sn XIV are present in band $135 \AA \pm 1 \%$ of available mirrors. Developers of the radiation source seek for increasing its efficiency by creating such specific plasma conditions which maximize intensity in the required band relatively that out of band. The most important is the long-wave region where radiation of lower ionization stages is present. Information on such spectra is either scarce or insufficiently reliable, requiring verification by comparison with isoelectronic spectra of neighbouring chemical elements, in the first turn, with indium spectra. Spectra of indium ions are interesting themselves for creation of a radiation source since a mixture of tin and indium is suggested for gain enhancing. Below is a progress report on the investigation of the five times ionized indium.

The spectrum of the five times ionized indium has been studied earlier elsewhere $[1,2]$. The ground state configuration of In VI is $4 p^{6} 4 d^{8}$. The transitions $4 d^{8}-4 d^{7} 5 p$ and $4 d^{7} 5 s-$ $4 d^{7} 5 p$ were investigated. The energy levels of the all three relevant configuration were established. Our project presumes a study of the other class of transitions, namely $4 p^{6} 4 d^{8}$ $\left(4 p^{5} 4 d^{9}+4 p^{6} 4 d^{7} 4 f+4 p^{6} 4 d^{7} 5 f+4 p^{6} 4 d^{7} 6 p\right)$. Exactly this class of transitions in ions of higher ionization stages having large transition probabilities creates in tin in band radiation.

The indium spectrum in the region $160-230 \AA$ (where the mentioned transitions are expected according to calculations) was obtained using the grazing-incidence spectrograph (incidence angle $85^{\circ}$, grating radius $3 \mathrm{~m}, 3600$ lines $/ \mathrm{mm}$ ). Spectra were excited in a vacuum spark with varied voltage $(1-5 \mathrm{kV})$, capacity $(12$ or $150 \mu \mathrm{F})$ and inductance $(100-930$ $\mathrm{nH})$, and as a result the peak current $(12-35 \mathrm{kA})$ for differentiation of spectral lines on ionization stages. Spectra were recorded on FUJI Imaging Plates. The latter were digitized on the Typhoon FLA 9500 scanner. The high linearity of imaging plates compared with

\footnotetext{
*Corresponding author: kononov@isan.troitsk.ru
} 
photographic plates allowed more reliable measurements of line intensity changes when spark discharge conditions were varied.

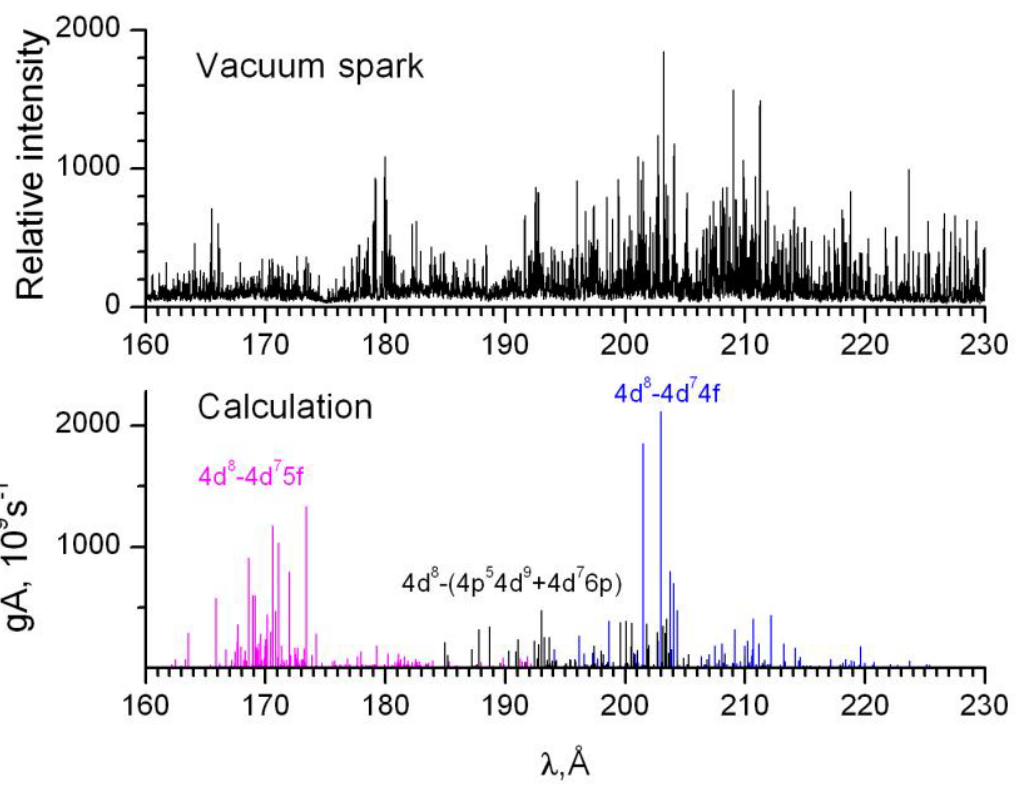

Fig. 1. Vacuum spark spectrum of indium and calculated transition probabilities in In VI.

The upper part of Fig. 1 shows an indium spectrum at peak current $12 \mathrm{kA}$. The lower part presents calculated transition probabilities (gA) in In VI. They were calculated by the Hartree-Fock method with relativistic corrections and semi-empirical scaling of energy parameters. The scaling factors were derived from the Sn VII isoelectronic spectrum.

More than 1600 spectral lines were measured on the spectrograms, among them more than 200 were attributed to In VI due to variations of their intensities with discharge conditions in the spark. A preliminary identification of the $4 d^{8}-4 d^{7} 4 f$ and $4 d^{8}-4 d^{7} 5 f$ transitions have been made.

The work was supported by the Russian Foundation for Basic Research, project № 1652-45036 IND_a.

\section{References}

1. V.S. Kushawaha, Y.N. Joshi, JOSA 66, 630 (1976)

2. Th.A.M. Van Kleef, Y.N. Joshi, JOSA 72, 1348 (1982) 